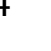

\title{
Antimicrobial properties of derivatives of the cationic tryptophan-rich
} hexapeptide PAF26

\author{
Running title: Antimicrobial properties of derivatives of PAF26
}

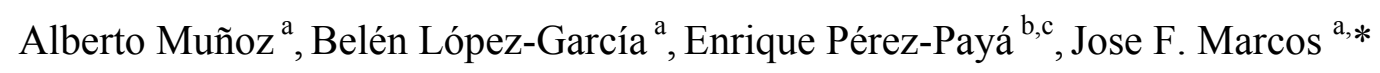

a Instituto de Agroquímica y Tecnología de Alimentos (IATA) - CSIC, Apartado de Correos 73, Burjassot, 46100 Valencia, Spain.

b Centro de Investigación Príncipe Felipe (CIPF), Avda. Autopista del Saler 16, 46013 Valencia, Spain.

c Instituto de Biomedicina de Valencia (IBV) - CSIC, Calle Jaime Roig 11, 46010 Valencia, Spain.

\footnotetext{
* Corresponding author:
}

Dr. Jose F. Marcos

$$
\begin{aligned}
& \text { Instituto de Agroquímica y Tecnología de Alimentos (IATA). } \\
& \text { Apartado de Correos 73. Burjassot. } 46100 \text { Valencia. Spain. }
\end{aligned}
$$$$
\text { e-mail: } \quad \text { jmarcos@iata.csic.es }
$$$$
\text { Tel: } \quad 34-96-3900022
$$

Fax: 34-96-3636301 


\section{$2 \quad$ Abstract}

Short antimicrobial peptides represent an alternative to fight pathogen infections. PAF26 is a hexapeptide identified previously by a combinatorial approach against the fungus Penicillium digitatum and shows antimicrobial properties towards certain phytopathogenic fungi. In this work, PAF26 was used as lead compound and its properties were compared with two series of derivates, obtained by either systematic alanine substitution or $\mathrm{N}$-terminal amino acid addition. The alanine scan approach underlined the optimized sequence of PAF26 in terms of potency and permeation capability, and also the higher contribution of the cationic residues to these properties.

11 The N-terminal addition of amino acids resulted in new heptapeptides with variations in

12 their antimicrobial characteristics, and very low cytolysis to human red blood cells.

13 Positive (Arg or Lys) and aromatic (Phe or Trp) residue addition increased broad

14 spectrum activity of PAF26. Noteworthy, addition of selected residues had specific

15 effects on the properties of derivatives of PAF26.

\section{$17 \quad$ Keywords}

18 Tryptophan-rich cationic antimicrobial peptide; AMP; antifungal peptide;

19 phytopathogenic fungi; $P$. digitatum; B. cinerea; $M$. grisea; F. oxysporum 
Introduction

Antimicrobial peptides (AMP) are important components of an evolutionarily ancient mechanism of immunity, found in a wide range of organisms [1]. AMP differ in length, sequence and structure, but generally are amphipathic and a great number have positive charge and are refereed as cationic antimicrobial peptides (CAMP). In many examples, these peptides are effective against microorganisms resistant to antibiotics or fungicides. In addition, AMP are unlikely to cause rapid emergence of resistance [2]. These facts and their short length, fast and efficient action against microbes and low toxicity to mammal cells have made them potential candidates as peptide drugs.

Rational design of AMP is an attractive approach to the improvement of antimicrobial properties. Agriculture could also greatly benefit from this emerging research area, with the identification, design and selection of peptides targeted to specific plant protection problems [3-6]. Soluble combinatorial libraries (SCL) represent an extensive source of molecular diversity for the de novo identification of lead AMP with new properties [7]. SCL have been used to identify novel peptides towards phytopathogenic fungi such as 66-10 hexapeptide (Ac-frlkfh- $\left.\mathrm{NH}_{2}\right)$ [8] and its derivative heptapeptide 77-3 (Ac-frlkfhf- $\mathrm{NH}_{2}$ ), which has activity against fungal strains of Fusarium sambucinum that are resistant to the fungicide thiabendazole (TBZ) [5]. In a previous work, we have used a synthetic D-hexapeptide library in a positional scanning format to identify AMP against selected phytopathogenic fungi that cause postharvest decay in fruits, such as Penicillium digitatum [6]. One of these peptides is PAF26 (Table 1), which showed strong activity against certain filamentous fungi and lower toxicity to Escherichia coli and Saccharomyces cerevisiae [6].

PAF26 is a tryptophan-rich CAMP with sequence similarities to other AMP [913]. It shares some properties with similar peptides, as absence of hemolytic activity $[12,14]$. PAF26 is active against strains resistant to fungicides and performed better than TBZ in experimental fruit decay tests [15]. Additionally, we have also demonstrated that PAF26 belongs to the class of AMP endowed with cell-penetrating properties $[16,17]$, being capable to specifically interact with and locate inside target fungal cells [14]. PAF26 and similar peptides synthesized with either D- or L-enantiomers do not differ substantially in antimicrobial potency $[9,11,14]$, which makes biotechnological production feasible. 
In this work, we have used PAF26 as a lead in an optimization strategy to design two sets of peptides with single residue variations. The purpose was to analyze the effect of such variations in the antimicrobial properties of the resulting peptides. First, alanine substitution analogues addressed the influence of each residue on PAF26 antimicrobial properties. Secondly, we designed and compared novel heptapeptides obtained by addition of different N-terminal residues to PAF26 in terms of (i) spectrum of activity, (ii) specificity, (iii) microbicidal properties, and (iv) cytolysis of human red

\section{Materials and methods}

Microorganisms. We used microorganisms that included fungal isolates of agricultural relevance (three distinct species of Penicillium, and Alternaria sp., $F$. oxysporum, B. cinerea and $M$. grisea) as well as fungal ( $A$. nidulans), yeast (S. cerevisiae) and bacterial (E. coli and B. subtillis) model strains (see Supplemental Table 4). Fungi were cultured on potato dextrose agar (PDA) (Difco-BD Diagnostics, Sparks, MD) plates at $24{ }^{\circ} \mathrm{C}$ with the exception of $M$. grisea, which was maintained on rice flour medium. Conidia were collected and adjusted to the appropriate

Peptides. Peptides used in this work (Table 1 and Supplemental Table 3) were concentration. S. cerevisiae was grown in YPD (1\% yeast extract, $1.5 \%$ peptone, $2 \%$ dextrose) at $30{ }^{\circ} \mathrm{C}$ and bacteria were grown in Luria-Bertani (LB) medium at $37^{\circ} \mathrm{C}$. purchased at $>90 \%$ purity (GenScript Corporation, Piscataway, NJ). Peptides were acetylated at the $\mathrm{N}$-terminus (Ac) and amidated at the C-terminus $\left(\mathrm{NH}_{2}\right)$. Stocks were prepared at $1 \mathrm{mM}$ in $5 \mathrm{mM} 3-(\mathrm{N}$-morpholino)-propanesulfonic acid pH 7 buffer and

Growth inhibition assays. The antimicrobial activities of the peptides were determined using a microtiter plate assay $[6,18]$. Growth was quantified as optical density (OD) at $492 \mathrm{~nm}$. Potato dextrose broth (PDB) (Difco-BD Diagnostics) diluted one twentieth (5\% PDB) was used as growth medium for fungi, and YPD diluted one tenth $(10 \%$ YPD) for yeast, in both cases containing $0.003 \%(\mathrm{w} / \mathrm{v})$ chloramphenicol. In 
antibacterial assays, the medium was LB diluted one tenth $(10 \% \mathrm{LB})$. Three replicates were prepared for each treatment.

The minimum inhibitory concentration (MIC) of a peptide for a given microorganism was the lowest peptide concentration that showed no growth at the end of the experiment. $\mathrm{The} \mathrm{IC}_{50}$ of a peptide was the concentration required to obtain $50 \%$ inhibition of growth, and the value in each experiment was estimated by adjustment of the experimental data (SigmaPlot v 8.02, SPSS Inc., Chicago, IL). Statistical analyses

Membrane permeation assays. Membrane permeation was determined with the probe Sytox Green (SG) (Molecular Probes-Invitrogen Corp., Carlsbad, CA) and were carried out with the software package StatGraphics Plus 4.0 (Manugistics Inc., Rockville, MD).

(1)

$$
\text { fluorometric measurement with a microplate reader (Fluoroskan Ascent FL, }
$$$$
\text { Labsystems, Finland) at an excitation of } 485 \mathrm{~nm} \text { and emission of } 538 \mathrm{~nm} \text { wavelengths }
$$
[14]. Three replicates were prepared for each treatment. The $\mathrm{FC}_{50}$ of a peptide was defined as the concentration inducing $50 \%$ of the maximum fluorescence emission, and the values were calculated by adjustment of the experimental data as above.

Fungicidal and bactericidal activity assays. Assessment of peptide microbicidal activity was conducted as follows. In the case of $P$. digitatum, $2.5 \times 10^{4}$ conidia/ml were incubated with peptides in $5 \% \mathrm{PDB}$ at $24{ }^{\circ} \mathrm{C}$. After 1 day of incubation, $50 \mu 1$ samples were spread onto peptide-free PDA plates to monitor colony forming units. S. cerevisiae and $E$. coli $\left(5.0 \times 10^{5} \mathrm{CFU} / \mathrm{ml}\right)$ were incubated for 1 day with peptides in either $10 \%$ YPD at $30^{\circ} \mathrm{C}$ or $10 \% \mathrm{LB}$ at $37^{\circ} \mathrm{C}$, respectively, and $2.5 \mu 1$ drops of samples were placed onto YPD or LB peptide-free plates. The lethal concentration (LC) of a peptide was defined as the lowest peptide concentration at which no growth or $<1 \%$ of CFU was recovered after peptide treatment. 


\section{Results and discussion}

Antimicrobial properties of a series of alanine substitution analogues of PAF26.

We designed a set of six Ala substitution analogues of the cationic tryptophanrich hexapeptide PAF26 (PAF26.r1a to PAF26.w6a, Table 1 and Supplemental Table 3). Distinct antimicrobial properties were determined and the results are summarized as $\mathrm{IC}_{50}$, MIC and LC towards P. digitatum (Table 2). We observed lower activity for all the analogues, although the decrease was higher in the peptides with substitution of the positively charged residues (PAF26.r1a, .k2a and .k3a), which approximately four-fold higher MIC and LC, and higher significant differences in the $\mathrm{IC}_{50}$.

We also used an assay based on the uptake of SG to quantify the permeation of establish a link between antimicrobial activity of AMP and cell permeation [5,19]. Previously, we demonstrated that incubation of fungal hyphae with PAF26 resulted in uptake and increase in the fluorescence of SG [14].

We have quantified and compared the permeation capability of the Ala analogues with PAF26, by determining permeation dose-response curves in conjunction with inhibition curves (Fig. 1). Data allowed the calculation of $\mathrm{FC}_{50}$ as an estimate of the permeation capability of peptides (Table 2). In the case of PAF26 and the PAF26.f5a analog, the permeation curve paralleled that of growth inhibition (Fig. 1A and $\mathrm{C}$ ), and both peptides had $\mathrm{IC}_{50}$ and $\mathrm{FC}_{50}$ values not significantly different (Table 2). Regarding the other five analogs, a noticeable result was the slight but consistently reproduced higher peptide concentrations needed to achieve $50 \%$ permeation $\left(\mathrm{FC}_{50}\right)$ than $50 \%$ inhibition $\left(\mathrm{IC}_{50}\right)$. This distinct effect could be visualized by plotting the relative activities for the six analogs as compared to PAF26 (Fig. 2). All the analogous except PAF26.f5a had losses of permeation capability (white bars) higher than losses of inhibition activity (black bars) (Fig. 2). Such differences among peptides are exemplified in a representative experiment (Fig. 1). PAF26.f5a initiates permeation and reaches maximum at concentrations similar to PAF26, in a curve that mirrors the inhibition response, while PAF26.rla has a permeation curve shifted to higher peptide concentrations as compared to growth inhibition. 
This differential effect of each Ala substitution in either the antimicrobial or the permeation properties dissociate to some extent growth inhibition from permeation among the different peptides, thus suggesting that PAF26 antimicrobial action is not solely based on its ability to permeate target cells. This has been previously proposed for other AMP $[1,2,9,16,17]$, and also explored in the case of PAF26, for which it was shown microscopically that produces growth alterations of mycelium in areas that are not permeabilized [14]. Moreover, PAF26 is internalized by $P$. digitatum hyphae at very low sub-inhibitory concentrations (i.e., $300 \mathrm{nM}$ ) that have no detectable effect on

(1)
growth, morphology or permeation [14].

Therefore, our Ala scanning approach showed that all the amino acid residues in PAF26 contribute to some extent to its antifungal or permeation activities and that none of them is dispensable for its properties towards $P$. digitatum. However, differences among the analogs were observed. PAF26 has an amphipathic arrangement with three $\mathrm{N}$-terminal cationic residues followed by three aromatic and hydrophobic residues at the C-terminus. Distinct independent parameters (Table 2 and Fig. 2) indicated that the antimicrobial potency and permeation properties were more affected by substitutions of the cationic rather than of the aromatic amino acids. The activity of CAMP, including those rich in Trp as PAF26, is dependent on the ionic environment [10,13,20], indicating that the initial interaction with microbes is electrostatic. In fact, confocal microscope observations have shown that indolicidin and PAF26 primarily interact with surfaces of hyphae $[13,14]$. The results of the Ala scan approach confirm the importance of such electrostatic interaction for antimicrobial activity and permeation.

Our data indicate that the two Trp residues follow in relevance to the cationic ones (Table 2). It has been reported that PAF26 interacts in vitro with membrane mimetics and that substitution of Trp-4 for Pro decreases this interaction and concomitantly also biological activity [21]. Finally, Phe-5 was found to be the least significant residue since its replacement produced a peptide with modest differences as compared to PAF26 and in fact its dose response curves were quite similar to that of PAF26 (Fig. 1).

Distinct activity profiles of heptapeptides derived from PAF26 by amino acid addition. Improvement in PAF26 antimicrobial potency and/or specificity could be achieved by means of replacement for, or addition of, specific amino acids. We have explored such scenario by addition of selected $\mathrm{N}$-terminal residues and screening of the 
1 resulting set of heptapeptides. Similar approaches have been used to improve other lead AMP [5,9]. Amino acids highly represented in AMP databases [22] were chosen: the positively charged residues Arg, Lys and His, the aromatic residues Trp, Phe and Tyr, the aliphatic residues Leu and Ala, and hydrophilic residues Thr and Gln (Table 1). The ten different resulting heptapeptides had 3-4 positive net charges at neutral $\mathrm{pH}$ and distinct hydropathic indexes that indicate a hydrophilic character (Supplemental Table 3).

An evaluation of antimicrobial potency and specificity was carried out against a panel of selected microorganisms that include fungi of agronomic relevance as well as the model filamentous fungus $A$. nidulans, the yeast $S$. cerevisiae, the Gram negative bacteria $E$. coli and the Gram positive $B$. subtillis, in order to assay overall activity,

2
specificity, and bioactivity against reluctant fungi. The parameters $\mathrm{IC}_{50}$ and $\mathrm{MIC}$ were

(1)
calculated (Supplemental Table 4). The previously described hexapeptide PAF34, which differ from PAF26 in two amino acid residues (Table 1), was introduced as a control of antimicrobial peptide with lower specificity than PAF26 [6].

Distinct inhibitory profiles of the heptapeptides were found (Supplemental Table 4). Considering the microorganisms, $P$. digitatum was the most sensitive to AMP based on the PAF26 lead, as expected given that this peptide was found in a combinatorial screen against this fungus. On the other hand, the least sensitive microorganisms were the phytopathogenic fungus $M$. grisea, the yeast $S$. cerevisiae and the bacteria $E$. coli. The non-filamentous microorganism that has the susceptibility pattern more similar to the filamentous fungi was the Gram positive bacterium B. subtilis.

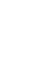

Taking into account the different peptides, the generally most active ones were PAF38 (Arg) and PAF39 (Lys), a result in agreement with the above demonstrated importance of cationic N-terminal residues in the activity of PAF26. However, our study found no clear correlation between the antimicrobial potency/specificity and the molecular weight, net charge, or hydrophilicity character of the peptides (Supplemental Table 4), suggesting that more complex interactions between the amino acid residues determine the antimicrobial properties. In other AMP, it has been shown that appropriate position/clustering of residues are determinant for activity and selectivity [12].

It was remarkable the higher activity against the economically important M. grisea of PAF41 (Phe) and PAF42 (Trp), but not of PAF38 and PAF39. In the case 
of $B$. cinerea and F. oxysporum a modest two-fold increase in the MIC was found in PAF40 (His) and PAF38, respectively, as compared to PAF26.

In most of the peptides the increase in antifungal activity correlates with an even higher increase in activity against the bacteria and yeast (Supplemental Table 4). In fact, the addition of cationic (PAF38, PAF39) or aromatic hydrophobic (PAF41, PAF42) residues showed a higher increase in antibacterial than in antifungal properties.

There were peptides that showed an improvement in the $\mathrm{IC}_{50}$ but a deleterious effect on the MIC, for instance in the case of PAF45 (Thr), PAF46 (Gln) or PAF47 (Ala) against $P$. digitatum, emphasizing the existence of differently shaped dose-

Microbicidal assays of heptapeptides derived from PAF26.

The killing capacity of peptides to conidia of P. digitatum, or cells of E. coli and S. cerevisiae was evaluated (Fig. 3). It has been previously shown that inhibitory and fungicidal properties against $P$. digitatum are not linked in selected AMP, as lactoferricin-derived peptides and melittin [14,18]. This experiment confirmed the response curves and the need for considering different parameters when characterizing antimicrobials [23]. reverse microbicidal properties of PAF26 and PAF34 (Fig. 3A). These two peptides have distinct activity profiles: PAF26 is more microbicidal than PAF34 to P. digitatum, while less to $S$. cerevisiae and E. coli.

Microbicidal data confirmed and extended the growth inhibition results described above. The cationic derivatives PAF38 and PAF39 showed a two-fold improvement of fungicidal activity against $P$. digitatum but also killed $S$. cerevisiae and E. coli more efficiently (Fig. 3B).

PAF40 has activity against $P$. digitatum and $S$. cerevisiae similar to the sequence-related PAF38, PAF39, PAF41 and PAF42, but a four-fold reduction in killing capacity to E. coli (Supplemental Table 4 and Fig. 3B). On the contrary, some of the peptides as PAF43 (Tyr) showed an increase in activity against bacteria while had activity against several fungi similar to PAF26 (Supplemental Table 4 and Fig. 3B). In fact, PAF40 and PAF43 showed reversal microbicidal properties towards $S$. cerevisiae and E. coli (Fig. 3B).

Also noticeable was the loss of fungicidal activity against conidia of $P$. digitatum of some peptides as PAF46 (Fig. 3B). We have reported that peptides derived from the antimicrobial motif of bovine Lactoferricin [10], which shown sequence 
1 similarities with PAF26 and also contain a Gln residue, have $\mathrm{IC}_{50}$ values very similar to PAF26 but a much lower fungicidal activity to $P$. digitatum [18]. This finding will guide future experiments to test whether Gln residues negatively impact fungicidal properties of CAMP while maintaining growth inhibition, and thus whether determinants of CAMP fungiestatic and fungicidal properties differ.

Overall, our results indicate that some of the modifications resulted in broader antimicrobial activity, and therefore would be undesirable whenever filamentous fungi are the specific target of the antimicrobial approach. Also, data provide information to the development of novel peptides with broader activities. The detrimental loss of antifungal properties of some heptapeptides also demonstrates that the presence of the PAF26 sequence by itself does not guarantee high inhibitory activity, confirming that a specific arrangement of a peptide sequence and interactions between residues are also

important for the properties of AMP [12,24].

\section{Evaluation of hemolytic activity of heptapeptides derived from PAF26.}

An evaluation of toxicity was performed by conducting hemolytic assays on human red blood cells at different peptide concentrations (1, 10 and $100 \mu \mathrm{M})$. All except one of the PAF26-derived peptides described in this work exhibited no hemolysis even at $100 \mu \mathrm{M}$ (i.e., below the detection limit, which in our assay is $0.3 \%$ of

\section{Conclusion} the hemolysis of the control). The only peptide showing marginal toxicity was PAF42 (Trp) that produced $0.6 \%$ hemolysis at $100 \mu \mathrm{M}$. The well known toxic peptide Melittin (used as control) was $100 \%$ hemolytic at $100 \mu \mathrm{M}$ and $38 \%$ at $10 \mu \mathrm{M}$. Data indicate that PAF heptapeptides are at least 1000 times less cytolytic to red blood cells than Melittin. It has been reported that PAF26 and Melittin are similarly active against $P$. digitatum, being Melittin more toxic to bacteria [6].

We have generated a series of peptides derived from PAF26, with different profiles of antimicrobial properties and negligible hemolysis. They are of interest in the development of novel AMP, adding to the catalog of compounds with potential application in agriculture and biomedicine. Selection of the most suitable peptide would depend on the importance for each particular use of potency and/or specificity. 


\section{Acknowledgements}

3

Work was supported by grants BIO2003-00927 and BIO2006-09523 from the

5 Spanish Ministry of Education and Science (MEC). B.L.-G. acknowledges the post-

6 doctoral program "Juan de la Cierva" of the Spanish MEC. We also acknowledge

7 M. José Pascual for her excellent technical assistance. 
Reference List

[1] K.A.Brogden, Antimicrobial peptides: Pore formers or metabolic inhibitors in bacteria?, Nat. Rev. Microbiol. 3 (2005) 238-250.

[2] M.R.Yeaman, N.Y.Yount, Mechanisms of antimicrobial peptide action and resistance, Pharmacol. Rev. 55 (2003) 27-55.

[3] C.Rudolph, P.H.Schreier, J.F.Uhrig, Peptide-mediated broad-spectrum plant resistance to tospoviruses, Proc. Natl. Acad. Sci. USA 100 (2003) 44294434.

[4] Z.D.Fang, J.G.Laskey, S.Huang, K.D.Bilyeu, R.O.Morris, F.J.Schmidt, J.T.English, Combinatorially selected defense peptides protect plant roots from pathogen infection, Proc. Natl. Acad. Sci. USA 103 (2006) 1844418449.

[5] C.F.Gonzalez, E.M.Provin, L.Zhu, D.J.Ebbole, Independent and synergistic activity of synthetic peptides against thiabendazole-resistant Fusarium sambucinum, Phytopathology 92 (2002) 917-924.

[6] B.López-García, E.Pérez-Payá, J.F.Marcos, Identification of novel hexapeptides bioactive against phytopathogenic fungi through screening of a synthetic peptide combinatorial library, Appl. Environ. Microbiol. 68 (2002) 24532460 .

[7] S.E.Blondelle, C.Pinilla, C.Boggiano, Synthetic combinatorial libraries as an alternative strategy for the development of novel treatments for infectious diseases, in: G.A.Morales, B.A.Bunin (Eds.), Methods in Enzymology: Combinatorial Chemistry, Part B,Academic Press, San Diego, 2003, pp. 322-344.

[8] J.D.Reed, D.L.Edwards, C.F.Gonzalez, Synthetic peptide combinatorial libraries: A method for the identification of bioactive peptides against phytopathogenic fungi, Molec. Plant-Microbe Interact. 10 (1997) 537-549.

[9] B.C.Monk, K.Niimi, S.Lin, A.Knight, T.B.Kardos, R.D.Cannon, R.Parshot, A.King, D.Lun, D.R.K.Harding, Surface-active fungicidal D-peptide inhibitors of the plasma membrane proton pump that block azole resistance, Antimicrob. Agents Chemother. 49 (2005) 57-70.

[10] D.I.Chan, E.J.Prenner, H.J.Vogel, Tryptophan- and arginine-rich antimicrobial peptides: Structures and mechanisms of action, Biochim. Biophys. Acta 1758 (2006) 1184-1202.

[11] M.Dathe, H.Nikolenko, J.Klose, M.Bienert, Cyclization increases the antimicrobial activity and selectivity of arginine- and tryptophancontaining hexapeptides, Biochemistry 43 (2004) 9140-9150. 
[12] A.Wessolowski, M.Bienert, M.Dathe, Antimicrobial activity of arginine- and tryptophan-rich hexapeptides: the effects of aromatic clusters, D-amino acid substitution and cyclization, J. Pept. Res 64 (2004) 159-169.

[13] D.G.Lee, H.K.Kim, S.A.Kim, Y.Park, S.C.Park, S.H.Jang, K.S.Hahm, Fungicidal effect of indolicidin and its interaction with phospholipid membranes, Biochem. Biophys. Res. Commun. 305 (2003) 305-310.

[15] B.López-García, A.Veyrat, E.Pérez-Payá, L.González-Candelas, J.F.Marcos, Comparison of the activity of antifungal hexapeptides and the fungicides thiabendazole and imazalil against postharvest fungal pathogens, Int. J. Food Microbiol. 89 (2003) 163-170.

[14] A.Muñoz, B.López-García, J.F.Marcos, Studies on the mode of action of the antifungal hexapeptide PAF26, Antimicrob. Agents Chemother. 50 (2006) 3847-3855.

[16] C.B.Park, H.S.Kim, S.C.Kim, Mechanism of action of the antimicrobial peptide buforin II: Buforin II kills microorganisms by penetrating the cell membrane and inhibiting cellular functions, Biochem. Biophys. Res. Commun. 244 (1998) 253-257.

[17] H.J.Jung, Y.Park, K.S.Hahm, D.G.Lee, Biological activity of Tat (47-58) peptide on human pathogenic fungi, Biochem. Biophys. Res. Commun. 345 (2006) 222-228.

[18] A.Muñoz, J.F.Marcos, Activity and mode of action against fungal phytopathogens of bovine lactoferricin-derived peptides, J. Appl. Microbiol. 101 (2006) 1199-1207.

[19] D.Rioux, V.Jacobi, M.Simard, R.C.Hamelin, Structural changes of spores of tree fungal pathogens after treatment with the designed antimicrobial peptide D2A21, Can. J. Botany 78 (2000) 462-471.

[20] B.López-García, L.González-Candelas, E.Pérez-Payá, J.F.Marcos, Identification and characterization of a hexapeptide with activity against phytopathogenic fungi that cause postharvest decay in fruits, Molec. PlantMicrobe Interact. 13 (2000) 837-846.

[21] B.López-García, J.F.Marcos, C.Abad, E.Pérez-Payá, Stabilisation of mixed peptide/lipid complexes in selective antifungal hexapeptides, Biochim. Biophys. Acta 1660 (2004) 131-137.

[22] C.Loose, K.Jensen, I.Rigoutsos, G.Stephanopoulos, A linguistic model for the rational design of antimicrobial peptides, Nature 443 (2006) 867-869.

[23] M.Rautenbach, G.D.Gerstner, N.M.Vlok, J.Kulenkampff, H.V.Westerhoff, Analyses of dose-response curves to compare the antimicrobial activity of model cationic alpha-helical peptides highlights the necessity for a minimum of two activity parameters, Analytical Biochemistry 350 (2006) 81-90. 
1 [24] K.Hilpert, M.R.Elliott, R.Volkmer-Engert, P.Henklein, O.Donini, Q.Zhou, D.F.H.Winkler, R.E.W.Hancock, Sequence requirements and an optimization strategy for short antimicrobial peptides, Chem. Biol. 13 (2006) 1101-1107. 


\section{Figure legends}

$3 \quad$ Fig. 1

4 Dose-response curves of inhibition (black circles) and permeation (white triangles) activity of peptides PAF26 (A), PAF26.r1a (B) and PAF26.f5a (C) on P. digitatum. Data shown are the mean values \pm SD of either OD $(492 \mathrm{~nm})$ or $(\mathrm{FI})(538 \mathrm{~nm})$.

Fig. 2

9 Relative antimicrobial and permeation activity of the Ala substitution analogs as 10 compared to the parental peptide PAF26. Bars represent the relative $\mathrm{IC}_{50}$ as measure of 11 growth inhibition (black bars), $\mathrm{FC}_{50}$ as measure of permeation (white bars), and $\mathrm{LC}$ as 12 measure of fungicidal activity to conidia (stripped bars). Relative values were calculated 13 taking the corresponding parameter of PAF26 as reference.

$15 \quad$ Fig. 3

16 Assessment of microbicidal activity of peptides against $P$. digitatum (left), S. cerevisiae 17 (middle) and E. coli (right). Microorganism samples, either conidia (P. digitatum) or $18 \mathrm{CFU}$ (S. cerevisiae and E. coli), were treated with selected peptide concentrations (top) 19 for 1 day, and spread (for the fungus) or applied as droplets (for the yeast and bacteria) 20 onto peptide-free plates. Representative photographs are shown for (A) the previously 21 described PAF26 and PAF34, and (B) for the novel heptapeptides described here 22 (PAF38 to PAF47). 
Table 1

Amino acid sequences of peptides.

\begin{tabular}{|c|c|}
\hline Peptide & Sequence $^{\mathrm{a}}$ \\
\hline PAF26 & $\mathrm{Ac}-r \mathrm{kkwfw}-\mathrm{NH}_{2}$ \\
\hline PAF34 & $\mathrm{Ac}-r \mathrm{kw} \mathbf{l} \mathrm{w}-\mathrm{NH}_{2}$ \\
\hline PAF26.r1a & $\mathrm{Ac}-\mathbf{a} k \mathrm{kwfw}-\mathrm{NH}_{2}$ \\
\hline PAF26.k2a & $\mathrm{Ac}-r \mathbf{a k w f w}-\mathrm{NH}_{2}$ \\
\hline PAF26.k3a & $\mathrm{Ac}-r \mathrm{kaw} \mathrm{fw}-\mathrm{NH}_{2}$ \\
\hline PAF26.w4a & $\mathrm{Ac}-r \mathrm{kk} \mathbf{a} \mathrm{fw}-\mathrm{NH}_{2}$ \\
\hline PAF26.f5a & $\mathrm{Ac}-r \mathrm{kkw} \mathbf{a} w-\mathrm{NH}_{2}$ \\
\hline PAF26.w6a & $\mathrm{Ac}-r \mathrm{kkwf} \mathbf{a}-\mathrm{NH}_{2}$ \\
\hline PAF38 & $\mathrm{Ac}-\boldsymbol{r} r \mathrm{kkwfw}-\mathrm{NH}_{2}$ \\
\hline PAF39 & $\mathrm{A}_{\mathrm{C}}-\mathbf{k} \mathrm{rkkwfw}-\mathrm{NH}_{2}$ \\
\hline PAF40 & $\mathrm{Ac}-\mathbf{h} r \mathrm{kkwfw}-\mathrm{NH}_{2}$ \\
\hline PAF41 & $\mathrm{AC}-\mathbf{f} r \mathrm{kkwfw}-\mathrm{NH}_{2}$ \\
\hline PAF42 & $\mathrm{AC}-\mathbf{w} r \mathrm{kkwfw}-\mathrm{NH}_{2}$ \\
\hline PAF43 & $\mathrm{Ac}-\mathbf{y} r \mathrm{kkwfw}-\mathrm{NH}_{2}$ \\
\hline PAF44 & $\mathrm{Ac}-1 \mathrm{rkkwfw}-\mathrm{NH}_{2}$ \\
\hline PAF45 & $\mathrm{Ac}-\mathrm{trkkwfw}_{\mathrm{N}} \mathrm{NH}_{2}$ \\
\hline PAF46 & $\mathrm{Ac}-\mathrm{q} r \mathrm{kkwfw}-\mathrm{NH}_{2}$ \\
\hline PAF47 & $\mathrm{Ac}-\mathbf{a r k k w f w}-\mathrm{NH}_{2}$ \\
\hline
\end{tabular}

${ }^{\text {a }}$ The D-amino acids are shown in lower case. Residues distinct from PAF26 are in bold. 
Table 2

Antimicrobial properties of PAF26 analogues towards P. digitatum.

\begin{tabular}{llccc}
\hline Peptide & $\mathrm{IC}_{50}(\mu \mathrm{M})^{\mathrm{a}, \mathrm{b}}$ & $\mathrm{MIC}(\mu \mathrm{M})$ & $\mathrm{LC}(\mu \mathrm{M})$ & $\mathrm{FC}_{50}(\mu \mathrm{M})^{\mathrm{a}, \mathrm{b}}$ \\
\hline PAF26 & $2.2 \pm 0.3(\mathrm{a})$ & 4 & 16 & $1.7 \pm 0.2(\mathrm{a})$ \\
PAF26.r1a & $6.3 \pm 1.1(\mathrm{c})$ & 16 & $>64$ & $11.5 \pm 1.1(\mathrm{c})$ \\
PAF26.k2a & $5.6 \pm 1.5(\mathrm{c})$ & 16 & 64 & $7.7 \pm 2.4(\mathrm{~b})$ \\
PAF26.k3a & $6.6 \pm 2.1(\mathrm{c})$ & 16 & $>64$ & $7.9 \pm 3.0(\mathrm{bc})$ \\
PAF26.w4a & $3.2 \pm 0.7(\mathrm{ab})$ & 8 & 32 & $6.3 \pm 1.4(\mathrm{~b})$ \\
PAF26.f5a & $2.8 \pm 0.6(\mathrm{ab})$ & 8 & 32 & $2.0 \pm 0.4(\mathrm{a})$ \\
PAF26.w6a & $3.7 \pm 0.5(\mathrm{~b})$ & 16 & 32 & $5.7 \pm 0.6(\mathrm{~b})$ \\
\hline
\end{tabular}

a Mean value \pm standard deviation, calculated from independent experiments.

b Values with the same letter do not differ at 95\% confidence level (Fisher's LSD procedure). 
Figure 1 (Muñoz et al., 2007)

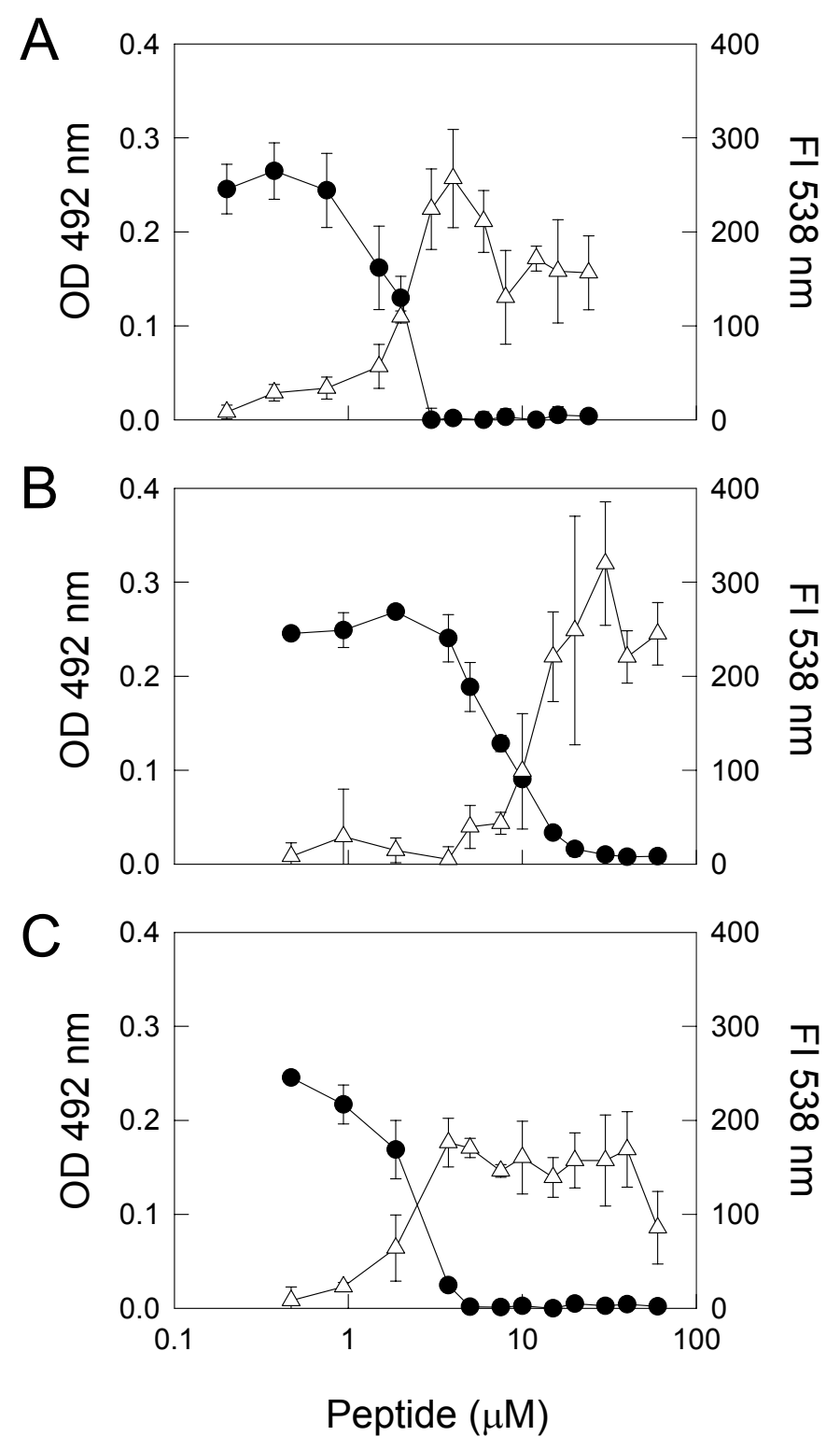


Figure 2 (Muñoz et al., 2007)

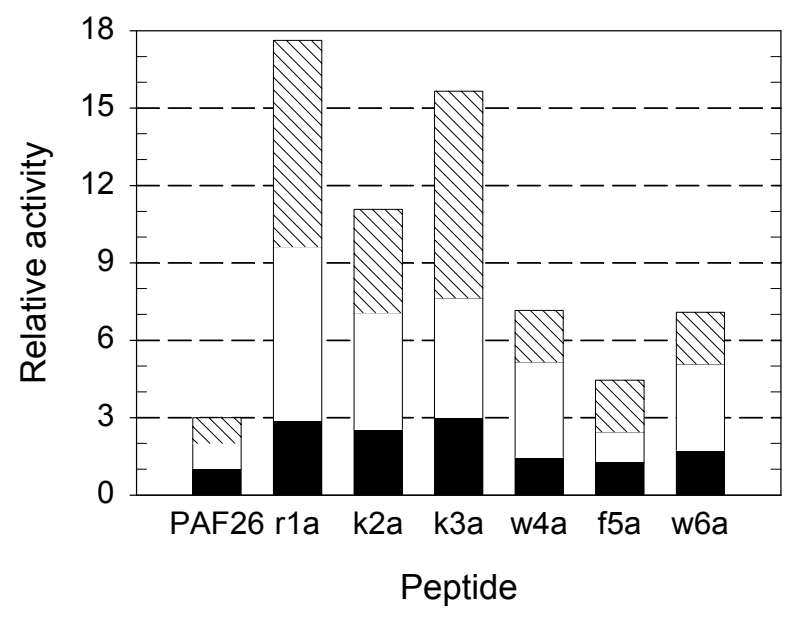


Figure 3 (Muñoz et al., 2007)
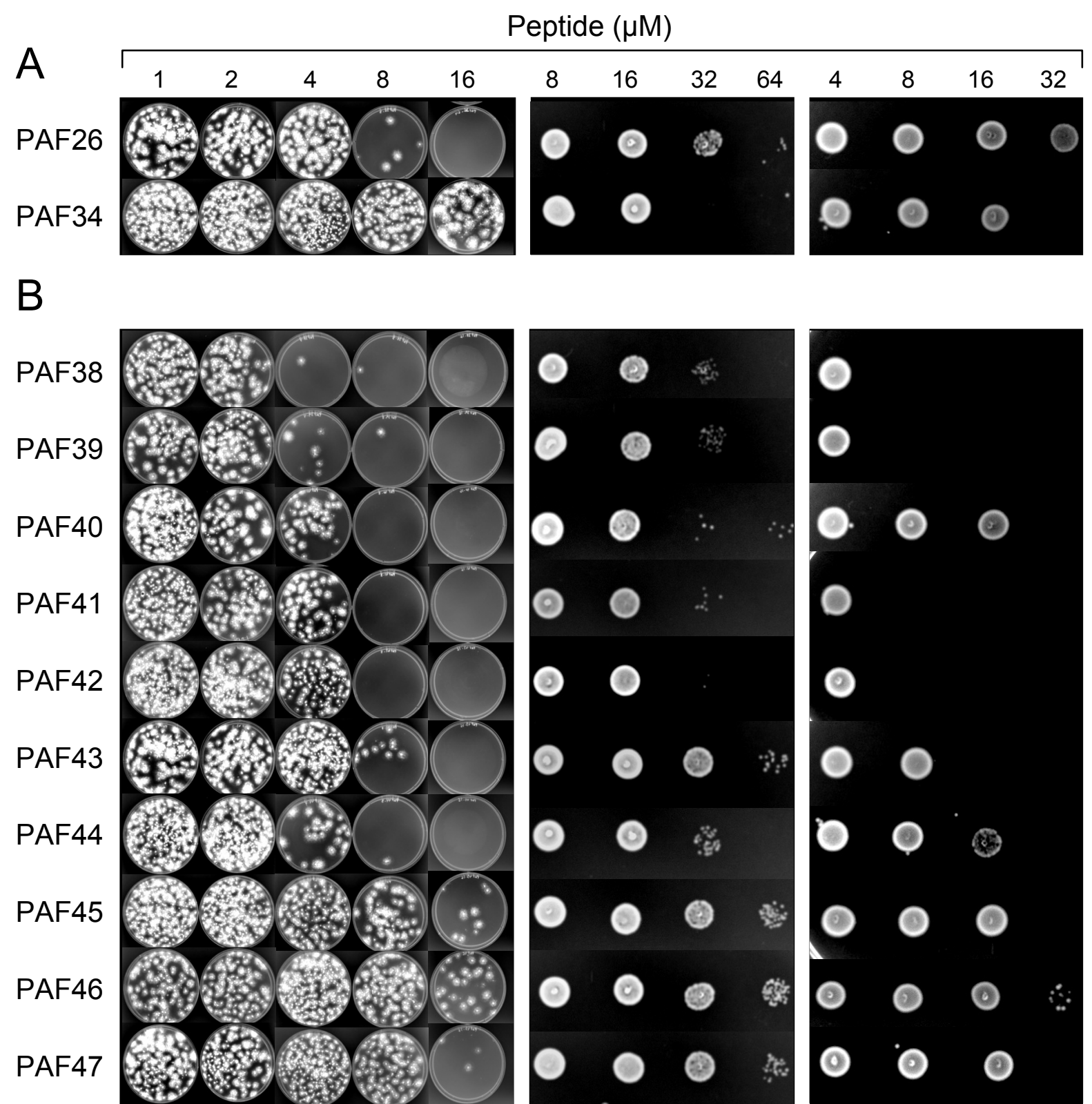

P. digitatum

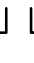

S.cerevisiae

E.coli 
Supplemental Table 3

Amino acid sequences and properties of peptides used in this study.

\begin{tabular}{|c|c|c|c|c|c|}
\hline Peptide & Sequence $^{\mathrm{a}}$ & MW & Net charge ${ }^{b}$ & GRAVY $^{\mathrm{c}}$ & Source \\
\hline PAF26 & $\mathrm{Ac}-r \mathrm{kkwfw}-\mathrm{NH}_{2}$ & 991.2 & $3+$ & -1.883 & {$[1]$} \\
\hline PAF34 & $\mathrm{Ac}-r \mathrm{kw} \mathbf{l} \mathrm{w}-\mathrm{NH}_{2}$ & 976.2 & $2+$ & -0.600 & {$[1]$} \\
\hline PAF26.r1a & $\mathrm{Ac}-\mathbf{a} \mathrm{kkwfw}-\mathrm{NH}_{2}$ & 906.1 & $2+$ & -0.833 & This study \\
\hline PAF26.k2a & $\mathrm{Ac}-\mathrm{rakwfw}-\mathrm{NH}_{2}$ & 934.1 & $2+$ & -0.933 & This study \\
\hline PAF26.k3a & $\mathrm{Ac}-r \mathrm{k} \mathbf{a} \mathrm{w} f \mathrm{w}-\mathrm{NH}_{2}$ & 934.1 & $2+$ & -0.933 & This study \\
\hline PAF26.w4a & $\mathrm{Ac}-\mathrm{rkk} \mathbf{a} \mathrm{fw}-\mathrm{NH}_{2}$ & 876.1 & $3+$ & -1.433 & This study \\
\hline PAF26.f5a & $\mathrm{Ac}-r \mathrm{kkw} \mathbf{a} w-\mathrm{NH}_{2}$ & 915.1 & $3+$ & -2.050 & This study \\
\hline PAF26.w6a & $\mathrm{Ac}-r \mathrm{kkwf} \mathbf{a}-\mathrm{NH}_{2}$ & 876.1 & $3+$ & -1.433 & This study \\
\hline PAF38 & $\mathrm{Ac}-\mathbf{r} r \mathrm{kkwfw}-\mathrm{NH}_{2}$ & 1147.4 & $4+$ & -2.257 & This study \\
\hline PAF39 & $\mathrm{Ac}-\mathbf{k} r \mathrm{kkwfw}-\mathrm{NH}_{2}$ & 1119.4 & $4+$ & -2.171 & This study \\
\hline PAF40 & $\mathrm{Ac}-\mathbf{h} r \mathrm{kkwfw}-\mathrm{NH}_{2}$ & 1128.3 & $3.1+$ & -2.071 & This study \\
\hline PAF41 & $\mathrm{Ac}-\mathbf{f} r \mathrm{kkwfw}-\mathrm{NH}_{2}$ & 1138.4 & $3+$ & -1.214 & This study \\
\hline PAF42 & $\mathrm{Ac}-\mathbf{w r k k w f w}-\mathrm{NH}_{2}$ & 1177.4 & $3+$ & -1.743 & This study \\
\hline PAF43 & $\mathrm{Ac}-\mathbf{y} r \mathrm{kkwfw}-\mathrm{NH}_{2}$ & 1154.4 & $3+$ & -1.800 & This study \\
\hline PAF44 & $\mathrm{Ac}-1 \mathrm{rkkwfw}-\mathrm{NH}_{2}$ & 1104.4 & $3+$ & -1.071 & This study \\
\hline PAF45 & $\mathrm{Ac}-\mathrm{trkkwfw}_{\mathrm{w}}-\mathrm{NH}_{2}$ & 1092.3 & $3+$ & -1.714 & This study \\
\hline PAF46 & $\mathrm{Ac}-\mathbf{q} \mathbf{r k k w f w}-\mathrm{NH}_{2}$ & 1119.3 & $3+$ & -2.114 & This study \\
\hline PAF47 & $\mathrm{Ac}-\boldsymbol{a r k k w f w}-\mathrm{NH}_{2}$ & 1062.3 & $3+$ & -1.357 & This study \\
\hline
\end{tabular}

${ }^{\text {a }}$ The D-amino acids are shown in lower case. Residues distinct from PAF26 are in bold.

${ }^{b}$ Estimated at $\mathrm{pH}=7$.

${ }^{c}$ Peptide grand average of hydropathicity (GRAVY) index [2] was calculated using a web-based tool (http://www.expasy.ch/).

\section{Reference List}

[1] B.López-García, E.Pérez-Payá, J.F.Marcos, Identification of novel hexapeptides bioactive against phytopathogenic fungi through screening of a synthetic peptide combinatorial library, Appl. Environ. Microbiol. 68 (2002) 2453-2460.

[2] J.Kyte, R.F.Doolittle, A simple method for displaying the hydropathic character of a protein, J. Molec. Biol. 157 (1982) 105-132. 
Supplemental Table 4

Growth inhibitory activity of synthetic peptides against different microorganism.

\begin{tabular}{|c|c|c|c|c|c|c|c|c|c|c|c|c|}
\hline \multirow{2}{*}{ Microorganism $^{a}$} & \multicolumn{12}{|c|}{ Peptide ${ }^{b}$} \\
\hline & PAF26 & PAF34 & PAF38 & PAF39 & PAF40 & PAF41 & PAF42 & PAF43 & PAF44 & PAF45 & PAF46 & PAF47 \\
\hline P. digitatum PHI-26 & $2.2(4)$ & $6.9(8)$ & $1.4(4)$ & $2.3(4)$ & $1.7(4)$ & $1.7(4)$ & $1.6(4)$ & $2.8(8)$ & $1.5(4)$ & $1.4(8)$ & $1.6(8)$ & $1.9(8)$ \\
\hline P. italicum PHI-1 & $2.3(4)$ & $6.0(8)$ & $1.9(4)$ & $3.4(8)$ & $2.1(4)$ & $2.4(4)$ & $3.4(4)$ & $2.1(4)$ & $2.2(4)$ & $2.2(4)$ & $3.2(8)$ & $3.8(8)$ \\
\hline P. expansum PHI-65 & $3.2(8)$ & $6.9(16)$ & $2.7(8)$ & $3.5(8)$ & $4.2(8)$ & $4.2(8)$ & $4.4(8)$ & $4.4(8)$ & $2.3(8)$ & $4.5(8)$ & $4.1(8)$ & $4.7(8)$ \\
\hline Alternaria sp. PHI-44 & $4.6(8)$ & $8.1(16)$ & $2.4(8)$ & $4.5(8)$ & $7.0(8)$ & $5.1(8)$ & $5.1(8)$ & $6.8(16)$ & $6.9(8)$ & $5.5(8)$ & $7.0(16)$ & $5.7(16)$ \\
\hline A. nidulans biA1 & $3.6(8)$ & $7.2(8)$ & $2.2(4)$ & $2.1(4)$ & $2.3(4)$ & $2.4(8)$ & $2.6(8)$ & $3.8(8)$ & $2.3(4)$ & $3.7(8)$ & $2.4(4)$ & $2.2(4)$ \\
\hline B. cinerea \#8 & $2.9(8)$ & $5.4(16)$ & $3.1(8)$ & $2.7(8)$ & $2.4(4)$ & $4.3(8)$ & $3.1(8)$ & $4.1(8)$ & $3.5(8)$ & $3.2(8)$ & $4.0(8)$ & $4.4(8)$ \\
\hline F. oxysporum CECT2866 & $4.5(8)$ & $7.5(16)$ & $2.3(4)$ & $2.3(8)$ & $2.5(8)$ & $2.7(8)$ & $2.4(8)$ & $4.1(8)$ & $3.7(8)$ & $4.1(8)$ & $3.4(8)$ & $4.1(8)$ \\
\hline M. grisea PR-9 & $(>32)^{\mathrm{c}}$ & $9.4(32)$ & $(>32)^{\mathrm{c}}$ & $(>32)^{\mathrm{c}}$ & $(>32)^{\mathrm{c}}$ & $9.0(16)$ & $6.7(16)$ & $(>32)^{\mathrm{c}}$ & $(>32)^{\mathrm{c}}$ & $(>32)^{\mathrm{c}}$ & $(>32)^{\mathrm{c}}$ & $(>32)^{\mathrm{c}}$ \\
\hline E. coli $\mathrm{DH} 5 \alpha$ & $20.4(64)$ & $15.5(32)$ & $4.5(8)$ & $6.7(8)$ & $12.7(32)$ & $4.5(8)$ & $5.1(8)$ & $8.7(16)$ & $14.4(16)$ & $17.1(32)$ & $22.2(64)$ & $17.3(32)$ \\
\hline B. subtilis CECT498 & $4.0(16)$ & $5.6(16)$ & $2.0(8)$ & $2.6(8)$ & $4.1(8)$ & $2.1(8)$ & $1.1(4)$ & $2.5(8)$ & $2.5(8)$ & $4.3(16)$ & $4.8(16)$ & $5.4(16)$ \\
\hline S. cerevisiae FY1679 & $15.9(32)$ & $13.4(32)$ & $9.0(16)$ & $8.1(16)$ & $9.2(16)$ & $10.5(16)$ & $16.6(32)$ & $17.6(32)$ & $15.5(32)$ & $13.0(32)$ & $12.9(32)$ & $20.1(32)$ \\
\hline
\end{tabular}

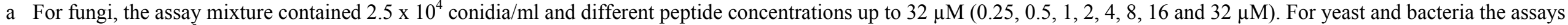
contained $5.0 \times 10^{5} \mathrm{CFU} / \mathrm{ml}$ and different peptide concentrations up to $64 \mu \mathrm{M}(2,4,8,12,16,24,32,48$ and $64 \mu \mathrm{M})$.

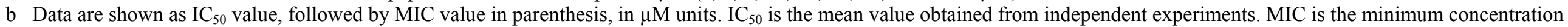
that was completely inhibitory in all the experiments.

c The symbol ">" indicates some, but not complete, inhibition of growth at the highest concentration used. In these cases, the $\mathrm{IC}_{50}$ was not calculated. 